\title{
Comparison of online, hands-on, and a combined approach for teaching cautery disbudding technique to dairy producers
}

\author{
Charlotte B. Winder, ${ }^{*}$ Stephen J. LeBlanc, ${ }^{*}$ Derek B. Haley, ${ }^{*}$ Kerry D. Lissemore, ${ }^{*}$ M. Ann Godkin, $\dagger$ \\ and Todd F. Duffield*1 \\ *Department of Population Medicine, University of Guelph, Ontario, N1G 2W1, Canada \\ †Ontario Ministry of Agriculture, Food, and Rural Affairs, Elora, Ontario, NOB 1S0, Canada
}

\begin{abstract}
The use of pain control for disbudding and dehorning is important from both an animal and industry perspective. Best practices include the use of local anesthetic, commonly given as a cornual nerve block (CNB), and a nonsteroidal anti-inflammatory drug. The proportion is decreasing, but many dairy producers do not use local anesthesia, perhaps in part due to lack of knowledge of the CNB technique. Although this skill is typically learned in person from a veterinarian, alternative methods may be useful. The objective of this trial was to determine if there were differences in the efficacy of online training $(\mathrm{n}=23)$, hands-on training $(\mathrm{n}=20)$, and a combined approach $(\mathrm{n}=23)$ for teaching producers to successfully administer a CNB and disbud a calf. The primary outcome was block efficacy, defined as a lack of established pain behaviors during iron application. Secondary outcomes were background knowledge (assessed by a written quiz), CNB and disbudding technique (evaluated by rubric scoring), time taken, and self-confidence before and after evaluation. Associations between training group and outcome were assessed with logistic regression, ordered logistic regression, and Cox-proportional hazard models, with a random effect for workshop. Block efficacy was not different between training groups, with $91 \%$ successful in both combined and online groups, and $75 \%$ in the hands-on trained group. Online learners had poorer technical scores than hands-on trainees. The combined group was not different from hands-on. Time to block completion tended to be longer for the online group (62 $\pm 11 \mathrm{~s}$ ), whereas time to disbudding completion was not different between hands-on (41 $\pm 5 \mathrm{~s})$ or combined trainees $(41 \pm 5 \mathrm{~s})$. The combined group had the highest pre-evaluation confidence score, and remained higher
\end{abstract}

Received May 23, 2017.

Accepted August 21, 2017.

${ }^{1}$ Corresponding author: tduffiel@uoguelph.ca after evaluation than online but was not different than hands-on. Although we saw some statistical differences between groups, absolute differences were small and block efficacy was similar. This suggests online training can be a useful tool for motivated producers who lack access to hands-on training.

Key words: disbudding, online learning, welfare, calves

\section{INTRODUCTION}

Almost all dairy operations in North America perform disbudding or dehorning (Adams et al., 2015) to ensure the safety of caregivers and other animals (Stock et al., 2013). On 75\% of farms these procedures are performed by the producer or farm staff, with the remainder using a veterinarian or veterinary technician (Winder et al., 2016). Cautery disbudding is used by 70 to $88 \%$ of dairy producers in North America (Vasseur et al., 2010; Adams et al., 2015; Winder et al., 2016).

Appropriate pain control for cautery disbudding involves the use of a local anesthetic, commonly given as a cornual nerve block (CNB) and a nonsteroidal antiinflammatory drug (Stafford and Mellor, 2011; Stock et al., 2013). Whereas administration of these medications in some countries requires veterinary supervision, in the United States and Canada it is common for these medications to be prescribed by the herd veterinarian for on-farm use. In a 2014 sample taken by the US National Animal Health Monitoring System, less than one third of producers used pain control for disbudding or dehorning (Adams et al., 2015). In Canada, use of pain control for dehorning is a requirement of the mandatory and enforced proAction Animal Care program, which began in September 2017 (Dairy Farmers of Canada, 2015). Although increasing industry pressure may be partly responsible for the apparent increase in use of local anesthetic in a recent survey in Ontario, $38 \%$ of dairy producers still do not use local anesthetic for these practices (Winder et al., 2016). Outside of program requirements, painful procedures are seen as a 
key welfare issue for the dairy industry by stakeholders (Robbins et al., 2015; Ventura et al., 2015).

Whereas local anesthetic is inexpensive, application of a CNB does require technical training on location of landmarks and injection technique to be successful. Lack of knowledge of how to perform this technique may partially explain why some producers do not adopt this form of pain control. Producers' perceived ability to implement a change is a key element to the adoption new management strategies (Ritter et al., 2017). This skill is typically acquired in person from a veterinarian, but several demonstration videos also exist online. Although a more personal approach can be tailored to an individual producer's needs and be more likely to result in motivating change (Ritter et al., 2017), not all producers have access to or desire individual training. To effectively reach all remaining dairy producers who do not currently provide local anesthesia for disbudding or dehorning, multiple forms of education and outreach may be beneficial. Eighty-nine percent of Canadian dairy producers reported accessing information for dairy farming through online resources (2014 Canadian National Dairy Study; D. Kelton, University of Guelph, Guelph, Ontario, unpublished data). Providing resources regarding appropriate administration of pain control for disbudding may be useful as a sole means of education, or a preliminary step to accessing individualized training from a veterinarian.

Our research group developed an interactive, online training module to teach how to administer a CNB, as well as properly disbud a calf using a small diameter thermal disbudding iron (Portasol, Elmira, OR). In a trial of veterinary students who had no prior experience giving a CNB, the online module was surprisingly effective, such that $75 \%$ of participants trained only online were able to administer an effective CNB without help (Winder et al., 2017). However, this was numerically less effective than the hands-on trained group $(100 \%$ success), and online-trained participants reported lower confidence and took more time to perform the technique (Winder et al., 2017).

The primary objective of the current study was to determine if an online training module was as effective as hands-on training for teaching dairy producers to successfully perform a CNB and disbud a dairy calf under 12 wk of age with a small diameter thermal disbudding iron (Portasol). Our hypothesis was that onlinetrained participants would be less successful compared with hands-on learning. A secondary objective was to determine if a combined approach of online and handson learning was more effective than hands-on training alone. We hypothesized that the combined approach would be more successful than hands-on training alone.

\section{MATERIALS AND METHODS}

Experimental methods were approved by both the University of Guelph Research Ethics Board's Certification of Ethical Acceptability of Research Involving Human Participants (REB\#16-12-618) and by the University of Guelph Animal Care Services (AUP\#3461). Calves in our study were housed on commercial farms as well as the University of Guelph Dairy Research and Innovation Center (Elora, Ontario, Canada). The study took place between January 2016 and January 2017.

\section{Sample Size}

Sample size was calculated with the primary outcome being CNB efficacy. Based on our similar trial with veterinary student participants (Winder et al., 2017), we estimated $70 \%$ success in the online-trained group and $95 \%$ success in the hands-on group, with a probability of type I error of 0.05 and power of 0.80 . Thus, we aimed to recruit 50 participants, with 25 in the online group and 25 in the hands-on group.

\section{Participants}

Five 1-d workshops were conducted for this trial. Members of the Ontario Association of Bovine Practitioners (Elora, Ontario, Canada) were contacted via email to recruit veterinary clinics interested in hosting a workshop on a client's farm. Two workshops were completed in which participants were recruited by their herd veterinarian. Three additional workshops were held at the University of Guelph's research dairy, where participants were recruited through a dairy producer magazine advertisement. Participants did not have experience performing a cornual block, which was confirmed when contact was made to provide details of the workshop.

\section{Demographic Information}

When participants arrived to the workshop, they were asked to complete a short written survey consisting of 4 questions with free-text responses. Questions included (1) Are you the person responsible for disbudding/dehorning calves on your farm; (2) What method is currently being used to disbud/dehorn calves; (3) At what age range are calves disbudded/dehorned on your farm; and (4) Why did you choose to attend today's workshop?

Responses to the question on reasons for attending the workshop were read by the first author (CBW) iteratively and coded, which were then sorted into themes. 


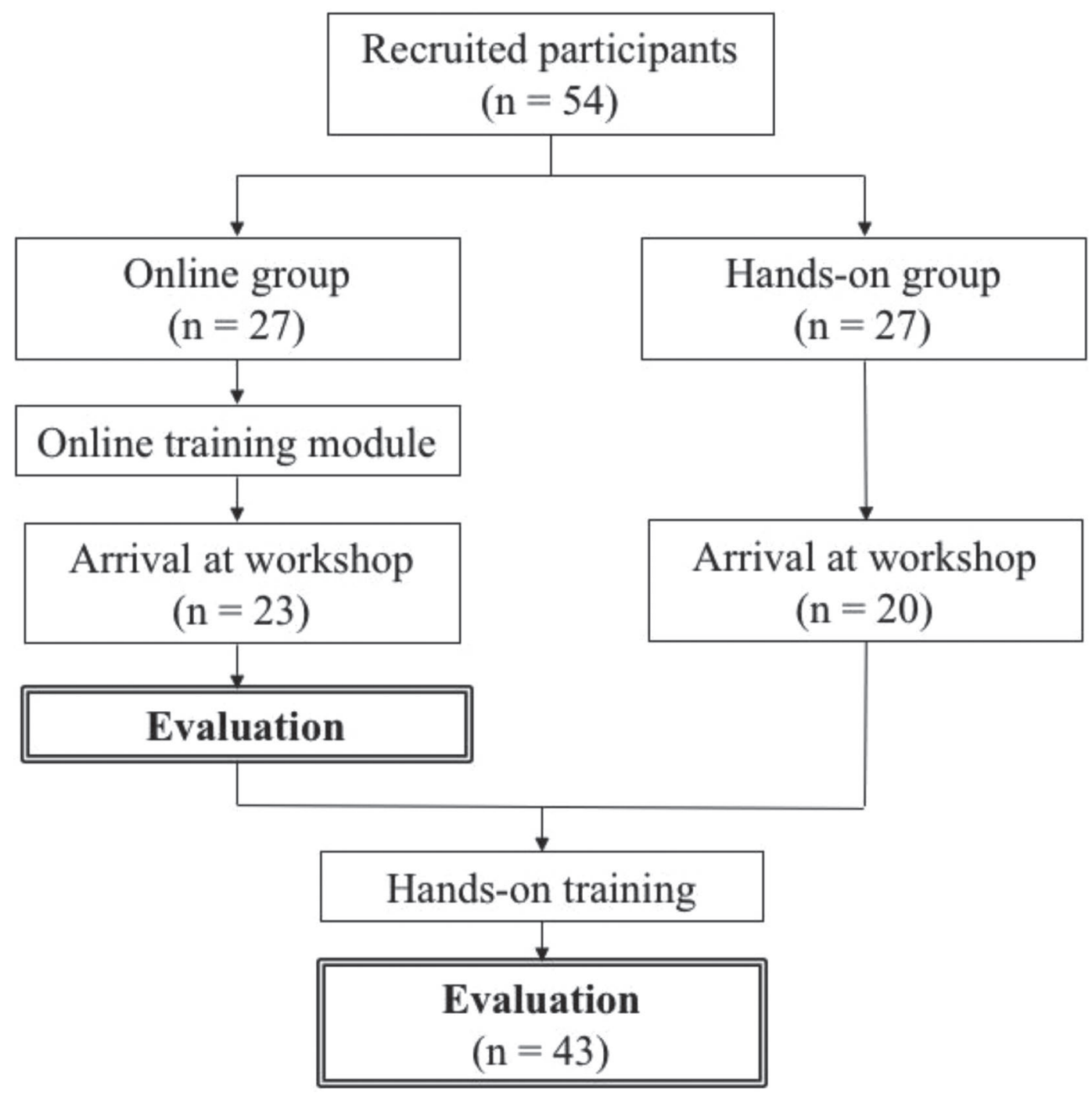

Figure 1. Flow of participants through the study of methods of training dairy producers to perform a cornual nerve block and disbud a calf less than 12 wk of age using a thermal disbudding iron (cautery).

Thematic analysis is a recognized analytic method for identifying patterns in qualitative data (Braun and Clark, 2006).

\section{Interventions}

Participant flow through the study is illustrated in Figure 1. Participants were assigned randomly to either online or hands-on training before the workshop date. Approximately 1 wk before the workshop, participants in the online group were given the uniform resource locator (URL) for an online training module to complete ahead of the workshop; those in the hands-on group were not given any training ahead of the workshop. Online-trained participants were evaluated separately at the beginning of the workshop, after which they completed the same hands-on training as the handon group. All participants were then evaluated after the hands-on training. Those in the online group thus served as 2 groups by completing 2 evaluations; one after online training only, and the other after they had also received hands-on training. The second evaluation of this group is referred to as the combined training group.

\section{Training and Evaluation Protocols}

Online training participants were given access to an online training module developed to mirror the handson training protocol. The training module included short videos with audio explanations, pictures, and an optional quiz. Participants were able to view the online 
module as many times as they wished before evaluation day. The module can be accessed online at http://bit .ly/disbudding (Articulate Storyline 2; Articulate, New York, NY).

Hands-on training was led by a registered veterinary technician for 4 workshops, and the first author (CBW) for 1 workshop. This followed a standardized protocol (see Supplemental File S1; https://doi.org/10.3168/ jds.2017-13217), in which there was an introductory discussion and a demonstration of both the CNB and disbudding technique on 1 calf ( 2 horn buds). Participants were then given a single horn bud each on which to practice, with as much help as they requested.

\section{Evaluations and Outcomes}

All evaluations were completed by the same evaluator, with each participant evaluated separately.

Primary Outcome. Efficacy of CNB was assessed during disbudding iron application, which occurred 10 min after CNB completion. A calf was considered to be inadequately blocked, and the participant was immediately stopped (rescued), if the calf performed any of the following behaviors during the first $5 \mathrm{~s}$ of the application of the dehorning iron: head shake, ear flick, foot stamp, vocalize, rear up, fall, kick, or tail flick. These have been used as behavioral indicators of disbudding and dehorning pain (Faulkner and Weary, 2000; Milligan et al., 2004; Heinrich et al., 2010) and were considered to indicate an inadequate local nerve block. Exceptions were made if it was clear the calf was avoiding flies. Rescued calves were then reblocked by the first author $(\mathrm{CBW})$ or the registered veterinary technician and the evaluation was continued. Rescues were also performed if any of the handling, restraint, or block techniques were considered to be unsafe for the calf or participant by the evaluator. Based on requirements from the Research Ethics Board, participants were also informed of their right to stop their participation in the study at any time if they wished. Anyone who declined to complete the evaluation were also to be considered to have been unsuccessful in block administration (rescued).

Secondary Outcomes. Evaluations included 4 sections: background knowledge, handling and restraint, CNB application, and disbudding iron application (see Supplemental File S1; https://doi.org/10.3168/jds .2017-13217). Background knowledge was assessed by a written quiz, which asked participants to identify the correct drug, volume, appropriate needle length and gauge, and appropriate injection site. Evaluations of calf handling, block application, and disbudding used rubric scoring. Handling and restraint score was based on correct application of the halter, choice of location to tie the calf, securing the calf's head tightly enough, and security of the quick-release knot. The CNB technique score was based on correct location and the number of needle insertions. Disbudding technique score was based on correct location and correct duration of application of the disbudding iron on the skin. Time taken for blocking and disbudding was also recorded, without the participants' knowledge. Participants were asked to rate their self-confidence in their ability to successfully block and disbud a calf on a scale of 1 (not confident) to 5 (very confident), both before and after the evaluation.

\section{Statistical Analysis}

Data were entered into Microsoft Excel (Microsoft Corp., Redmond, WA) and exported into STATA13 (Stata/IC Version 13.1 for Mac, StataCorp, College Station, TX). Variables were screened, and those with little variation $(<5 \%)$ were removed from further analysis. The effect of treatment group was determined for each outcome. Written quiz score, pre-evaluation confidence score, CNB administration technical score, disbudding technical score, and postevaluation confidence score were analyzed using multilevel ordered logistic regression models with a random effect for replicate. Model fit was assessed by examining standardized residuals and BLUP graphically for homoscedasticity and normality. Outliers were examined to ensure accuracy of data but were not excluded. A multilevel logistic model with a random effect for replicate (workshop) was used to determine the effect of treatment group on CNB efficacy. Model fit was assessed by examining homoscedasticity of Pearson residuals and homoscedasticity and normality of BLUP. The effect of treatment group on time to complete CNB administration and time to complete disbudding iron application was evaluated using Cox proportional hazard models with a fixed effect for study replicate. The hazard ratio was used to describe the risk of completion of a given step (cornual block administration or disbudding iron application) in a given time for one group compared with another. The proportional hazard assumption was assessed by the Kaplan-Meier graphs, and a nonsignificant test of nonzero slope by plotting Schoenfeld residuals over log of time. For all tests, statistical significance was defined as $P<0.05$ and tendencies at $P<0.10$.

\section{RESULTS}

Fifty-four participants agreed to enroll in a workshop ( 8 to 14 per workshop); however, due to weather condi- 
tions and on-farm commitments, 11 potential participants were unable to attend on the workshop day. All 43 participants who arrived on the workshop day (6 to 10 per workshop) completed the trial; no participants declined to fully complete the training and evaluation components they were assigned.

\section{Demographic Information}

Thirty-six participants chose to complete the demographic survey. Half stated they were the person currently responsible for disbudding or dehorning on their farm. The majority (28/36) used cautery disbudding, 3 used caustic paste disbudding, and 5 reported using surgical amputation dehorning (i.e., gouging). Mean minimum age for disbudding was 8 wk (range $=0-40$; $\mathrm{SD}=10$ ) and mean maximum age was $14 \mathrm{wk}$ (range $=$ 3-96; SD = 18). Seven themes emerged from examination of the free text responses to the question asking the reason for participation in the workshop. These themes consisted of wanting to learn or refine a new skill (16), concern for pain associated with disbudding (3), wanting to start disbudding themselves instead of using a veterinarian (7), wanting to start disbudding themselves instead of another staff member (2), being told to attend the workshop by their veterinarian (2), concern for proAction requirements (1), and the lunch that was provided at the workshop (1).

\section{Effect of Treatment Group on Outcomes}

Initial screening showed handling scores contained little variation; as only 2 participants did not achieve a maximum score, this variable was removed from further analysis. A full description of the evaluations is available in Supplemental File S1 (https://doi.org/10.3168/ jds.2017-13217).

CNB Efficacy. The primary outcome of CNB efficacy was not statistically different among groups, with the online-trained group having 91\% success, the hands-on group having $75 \%$ success, and the combined group having $91 \%$ success (combined vs. hands-on, $P$ $=0.17$; online vs. hands-on, $P=0.17$; combined vs. online, $P=1.00)$. Interestingly, all failures in the online and combined groups were unique participants; that is, those who had a CNB failure after online training (n $=2$ ) did not have a failure after additional hands-on training (combined group), and those who had a failure after combined training $(\mathrm{n}=2)$ previously had a successful CNB after online training alone.

Background Knowledge. Rubric scores by training group are summarized in Figure 2. Background knowledge was assessed by a written quiz out of 5 ; mean scores were significantly higher in the combined group $(4.9 \pm 0.1)$ compared with hands-on group $(4.4 \pm 0.2$, $P=0.03)$, whereas the online group was not different from either $(4.7 \pm 0.1)$. This model fit the data well.

Technical Scores. The CNB and disbudding technical scores were marked out of 3 . Mean CNB score was lowest in the online group $(2.3 \pm 0.2)$, with the hands-on group having a higher score $(2.6 \pm 0.1, P$ $=0.02$ ) that was not statistically different from the combined group $(2.5 \pm 0.1)$. A similar pattern was seen with disbudding score; the online group's mean score $(2.6 \pm 0.1)$ was significantly lower than hands-on $(2.9 \pm 0.1, P=0.02)$ and tended to be lower than the combined group's mean score $(2.8 \pm 0.1, P=0.08)$. The CNB technical score model's higher-order residuals followed a normal distribution but showed signs of heteroscedasticity (residuals were not similar across all predicted values of the model), whereas the disbudding technical score model fit the data well.

Confidence Scores. Self-reported confidence scores were defined as 5 being very confident and 1 being very unconfident with respect to their ability to effectively administer a CNB and disbud a calf. Differences in confidence score by training group are summarized in Figure 3. Pre-evaluation, the online group's mean score $(3.3 \pm 0.2)$ was lower than the hands-on group $(3.8 \pm 0.2, P=0.05)$. The combined group's mean pre-evaluation confidence score $(4.5 \pm 0.1)$ was significantly higher than both the hands-on $(P=0.01)$ and online groups $(P<0.001)$. After evaluation, all group mean scores were higher, with the online $(4.5 \pm 0.1)$ and hands-on group $(4.5 \pm 0.2)$ not different from each other $(P=0.13)$ but the combined group was higher than the online $(4.8 \pm 0.1, P=0.02)$; these models fit the data well.

Time Taken. Time to CNB completion is illustrated in Figure 4. Risk of completion over a given time was lower for the online group, with a hazard ratio of 0.6 $(95 \% \mathrm{CI}=0.3-1.1)$ compared with the hands-on group $(P=0.09)$, and a hazard ratio of $0.6(95 \% \mathrm{CI}=0.3-1.1)$ compared with the combined group $(P<0.05)$. Risk of completion did not differ from the hands-on group $(P=$ 0.78). Median time to completion for the online group was $62 \pm 11 \mathrm{~s}$, with an interquartile range (IQR) of 54 $\mathrm{s}$. The hands-on group took a median time of $41 \pm 5 \mathrm{~s}$ with an IQR of $17 \mathrm{~s}$, with the combined group taking a median time of $41 \pm 5 \mathrm{~s}$ and an IQR of $30 \mathrm{~s}$. Time to completion of disbudding iron application is illustrated in Figure 5. Risk of completion over a given time was not statistically different between groups. The online group took a median time of $76 \pm 11 \mathrm{~s}$ with an IQR of $112 \mathrm{~s}$, whereas the hands-on group had a median time of $48 \pm 6 \mathrm{~s}$ with an IQR of $54 \mathrm{~s}$ and the combined group 


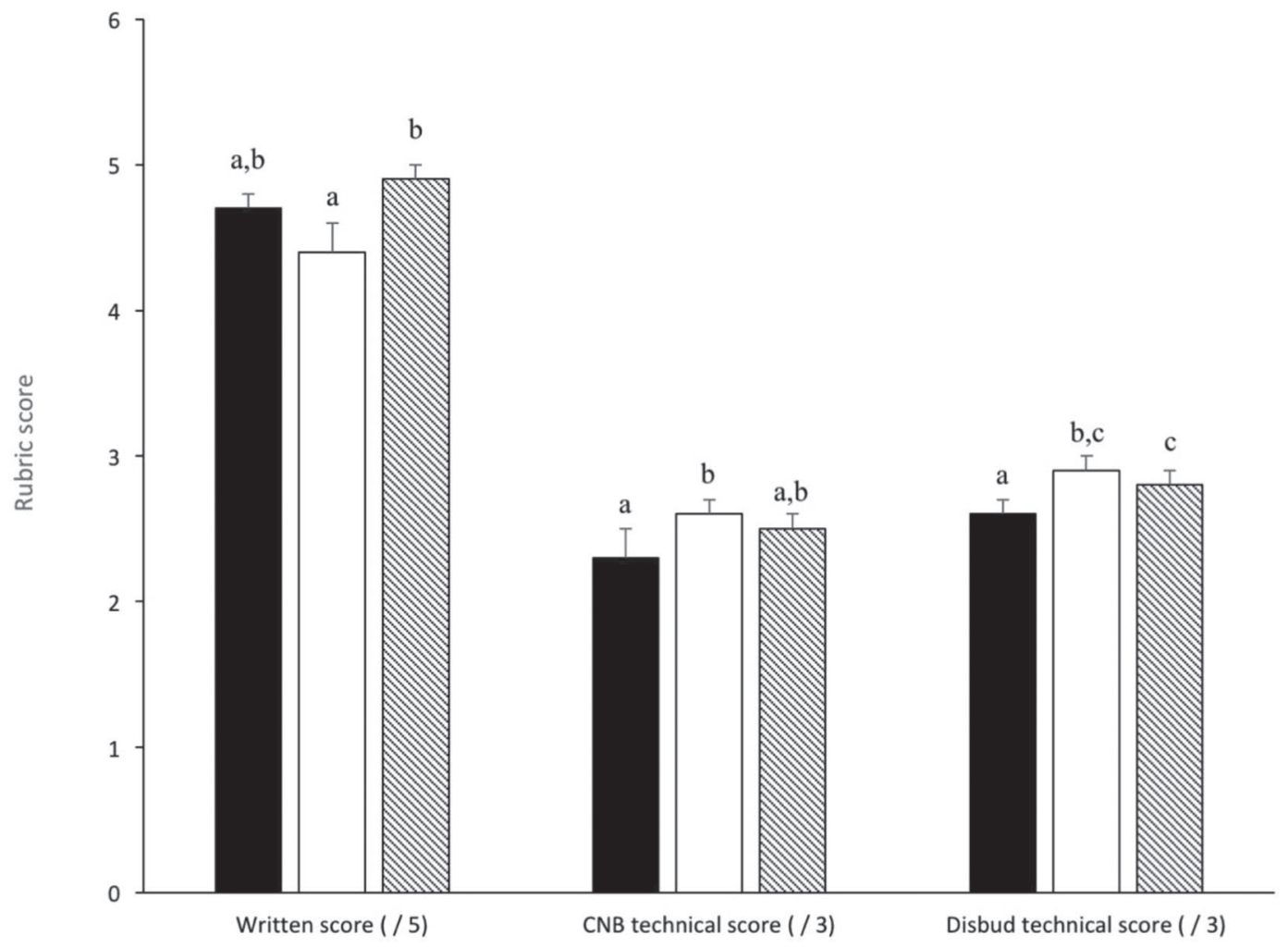

Figure 2. Rubric score with SE bars for the written quiz assessing background knowledge (out of 5), technical score for cornual nerve block (CNB) application (out of 3), and technical score for disbudding iron application (out of 3), by training group [online trained, closed bars; handson trained, open bars; combined group (online and hands-on), cross-hatched bars]. Letters a and b indicate $P<0.05$ between training groups for a given outcome, whereas $\mathrm{c}$ indicates $P<0.10$ between training groups for a given outcome.

had a median time of $65 \pm 8 \mathrm{~s}$ with an IQR of $87 \mathrm{~s}$. The proportional hazards assumption appeared valid for both models.

\section{DISCUSSION}

The primary difference seen between our study groups was reported self-confidence; whereas online learners were able to perform an effective block as well as other groups, they were less confident in their ability to do so. Therefore, although online learning shows promise as a tool to teach producers to perform a cornual nerve block, it should be noted that it does not instill the same degree of confidence as hands-on learning.

\section{Online Versus Hands-On Training}

The success of the cornual block in our online-trained group was unexpected. Although a similar finding was seen in our previous work with veterinary students (Winder et al., 2017), the hands-on group still had a numerically higher success rate (75 vs. $100 \%)$. In our current study, the online group had numerically more successes compared with the hands-on group (91 vs. $75 \%$ ). Despite equal likelihood of block success, online learners were less confident before evaluation, were less skilled in block application, and tended to take more time (an additional $20 \mathrm{~s}$ ) to perform the block. Without technical practice, it is unlikely online training alone would confer as much confidence for a psychomotor skill compared with training methods that encompass hands-on training. That said, motivated producers who learn this skill would typically have multiple calves available to practice their skills on, and the nature of the cornual block would ensure clear and immediate feedback on disbudding iron application, which should increase confidence in the majority of participants. As a result, we feel online training shows promise as a tool for motivated producers who lack access to hands-on training.

\section{Combined Training}

Although combined training was associated with better background knowledge and higher confidence before evaluation, neither block success nor time taken 


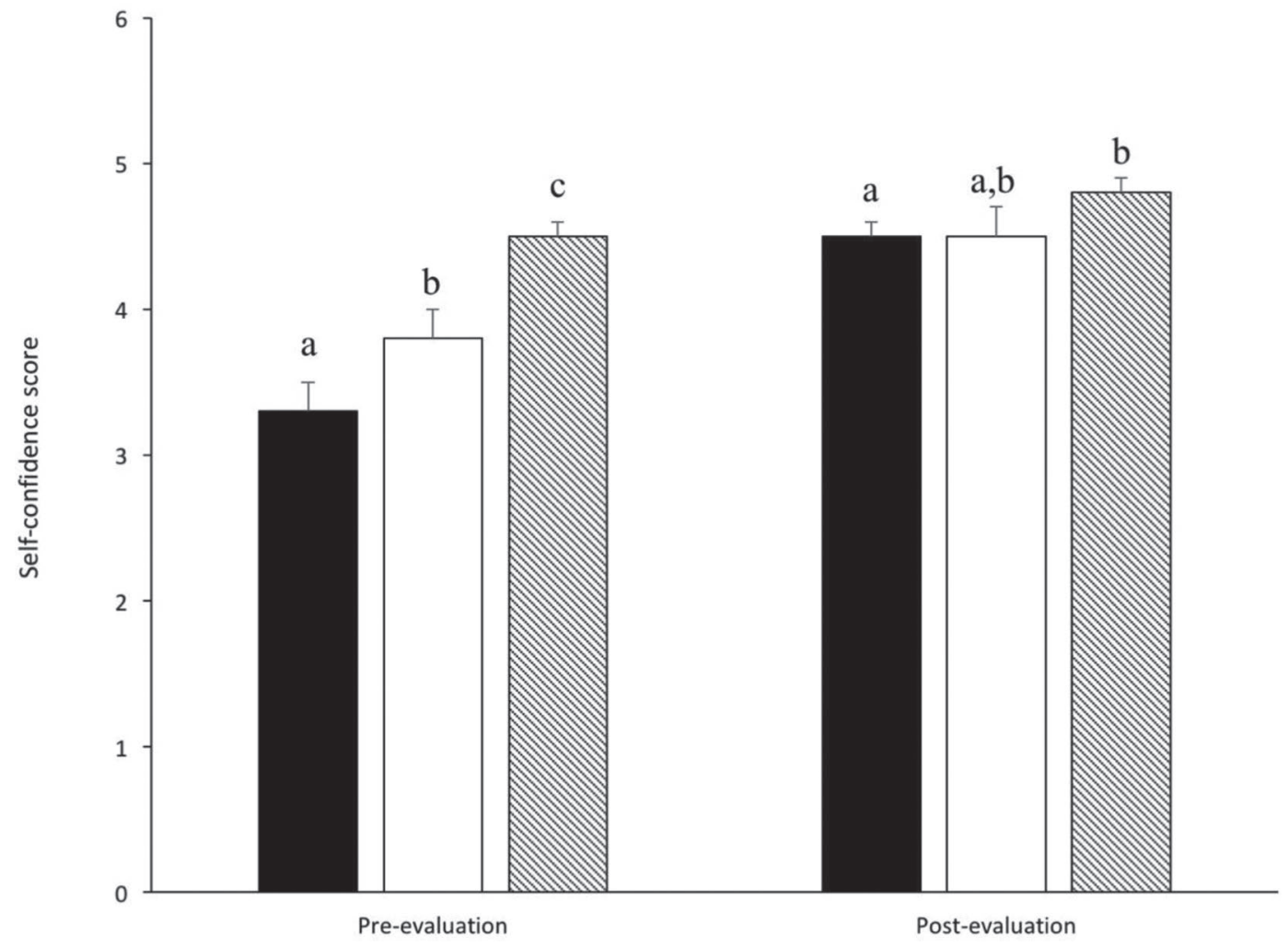

Figure 3. Self-assessed confidence score with SE bars (out of $5 ; 1=$ not confident and $5=$ very confident) by training group [online trained, closed bars; hands-on trained, open bars; combined group (online and hands-on), cross-hatched bars]. Letters a and $\mathrm{b}$ indicate $P<0.05$ between training groups for a given outcome, whereas c indicates $P<0.10$ between training groups for a given outcome.

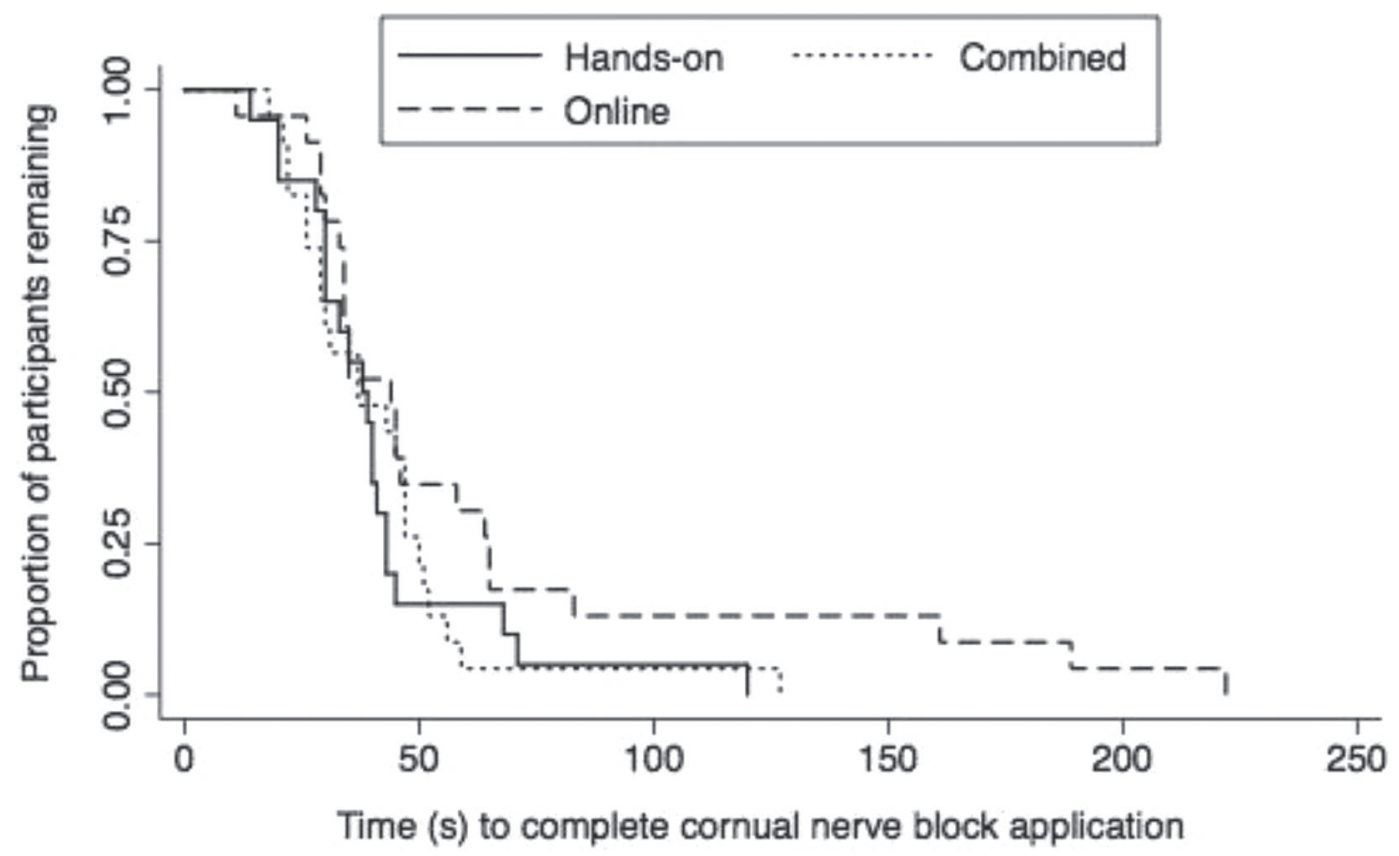

Figure 4. Kaplan-Meier survival function showing time to completion for administration of cornual nerve block for hands-on trained participants (solid line), online-trained participants (long dashed line), and those trained with a combined approach (short dashed line). 


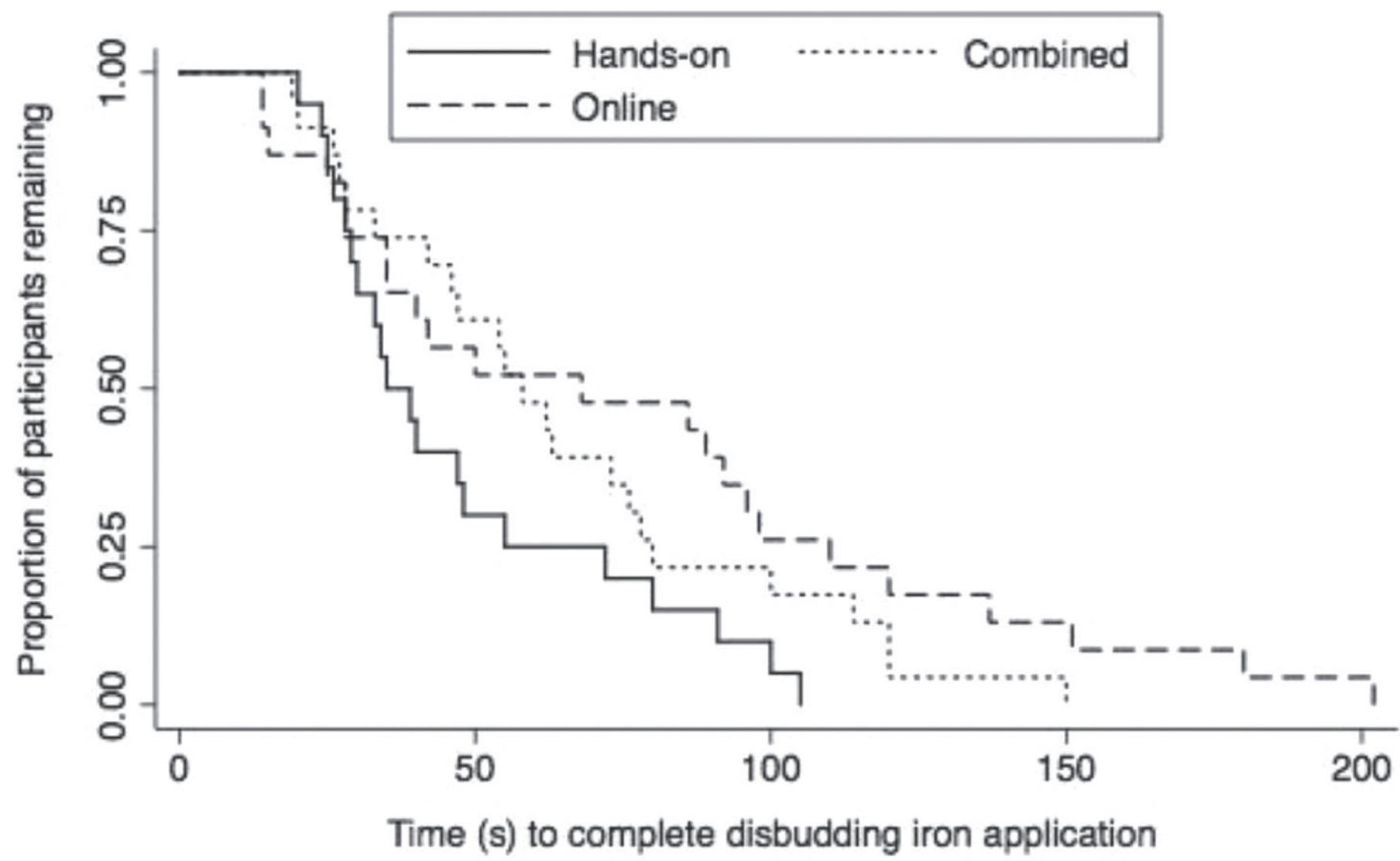

Figure 5. Kaplan-Meier survival function showing time to completion for application of disbudding iron for hands-on trained participants (solid line), online-trained participants (long dashed line), and those trained with a combined approach (short dashed line).

was different from the solely hands-on or online-trained groups, and differences in technical scores were slight over the online group, even though the written quiz was the same one completed after online training. This could indicate only a small benefit exists in the additional training, conceivably because many participants achieved maximum rubric scores there was little in our scoring system to evaluate differences between these groups. The increase in confidence could be due to the additional training, to the additional experience gained practicing on a calf, or both. It may also be in part due to familiarity with the evaluation procedure itself, as they had previously been evaluated after online training. More research is required to parse out these differences.

\section{Considerations}

Based on the design of the workshops, it was not possible to blind the evaluator to the treatment groups, and, as such, we attempted to make any subjective scoring as clear as possible by using a rubric (see Supplemental File S1; https://doi.org/10.3168/ jds.2017-13217). In addition, measuring time taken to complete each task and the participant's own evaluated self-confidence were used to further reduce potential bias from the evaluator.

Individual differences may have existed between calves, which would have made them more or less likely to have a successful block (tractability, biology, experience with a previously unsuccessful block, and so on), but the distribution of the calves throughout the trial was not a function of participant training group, with the exception that all evaluations of the online group were done first. Therefore, none of those calves would have experienced a previous unsuccessful block. However, these calves may have also been less familiar with the restraint in this group, whereas calves previously handled with a successful block could potentially be easier to handle.

Although it was necessary to provide a formal evaluation to judge the relative success of the training group, to an extent, the effect of the evaluation may have influenced the outcomes (Hawthorne's bias, the potential for behavioral change in response to being observed). Study participants may have felt more comfortable try- 
ing out a new skill in the presence of the evaluator, as they were required to be informed (as per the research ethics board) that they would be stopped if their actions were dangerous to either the calf or themselves. If the same participants were trying the technique on their own calves, they may have a more apprehensive approach, which could influence the effect. Conversely, it is also possible some participants could have felt intimidated by the evaluator and would be more comfortable trying a new skill on their own. Although producers who were recruited for our study were required not to be currently using a CNB for disbudding, they may not represent all producers. Therefore, the success of the online training module could in part be due to the inherent motivation to learn of the participants who volunteered.

As we did not achieve our sample size goal, it is possible that some numerical differences may have achieved statistical significance had there been a larger study population. However, the apparent difference was smaller than expected.

Internet usage is widespread among dairy producers (2014 Canadian National Dairy Study; D. Kelton, University of Guelph, Guelph, Ontario, unpublished data), but it should be noted that it is not universal, and broadband coverage is still lacking in some rural and remote communities in Canada (Canadian Radio-television and Telecommunications Commission, 2015). If these areas also reflect those underserviced by veterinarians, this may reduce the effect of the online module. However, federal efforts are underway to provide enhanced access to broadband internet in such communities (Canadian Radio-television and Telecommunications Commission, 2015).

\section{Potential Application}

Dairy producer's perceptions and motivation to adopt new management strategies can vary substantially, and different strategies are therefore needed (Ritter et al., 2016). Different personality types and learning styles may mean some producers will be more receptive to online learning, whereas others may prefer a different approach. To reach all nonadopters, especially those without a strong relationship with a veterinarian, the online learning module may have merit, including providing an avenue for producers who do not wish to or cannot access hands-on training; allowing producers to gain additional knowledge in conjunction with handson training; providing a resource for teaching a new farm employee the technique; and helping initiate discussion of disbudding pain control with other producers or their herd veterinarian.

\section{CONCLUSIONS}

Use of an online learning module resulted in a similar ability to provide an effective CNB when compared with a group receiving hands-on training, albeit with less confidence. A combined approach increased confidence. Marginal differences in skill level were seen between groups, with the hands-on and combined group having a small advantage over online training alone. Whereas best practices likely include one-on-one training with a veterinarian, to improve adoption of pain control for disbudding among dairy producers the promotion of an online module may be of benefit for those who are unable or unwilling to access hands-on training. Online learning alone may not be suitable for those lacking confidence to attempt a new technique without assistance, but may be valuable as an additional tool with hands-on training, to teach additional employees on a given farm, or as a way to foster discussion on pain control for disbudding.

\section{ACKNOWLEDGMENTS}

The authors thank Charlotte Friendship and Angela Runciman (Campbellford, Ontario); David Douglas, Jennifer Hubbard, and Kimberly Spence (Navan, Ontario); and Emilie Belage, Sarah Ferguson, Catalina Medrano Galarza, and Rachel Skippen (University of Guelph, Guelph, Ontario) for their valuable contributions to this work. In addition, this study would not have been possible without the indispensable participation of the producers who volunteered to attend the workshops. This project was funded by a Knowledge Translation and Transfer grant from the Agri-Food and Rural Link program of the Ontario Ministry of Agriculture, Food, and Rural Affairs - University of Guelph partnership (Guelph, Ontario, Canada).

\section{REFERENCES}

Adams, A. E., J. E. Lombard, C. S. Shivley, N. J. Urie, I. N. RomanMuniz, C. P. Fossler, and C. A. Kopral. 2015. Management practices that may impact dairy heifer welfare on U.S. dairy operations. J. Dairy Sci. 98(Suppl. 2):105. (Abstr.)

Braun, V., and V. Clark. 2006. Using thematic analysis in psychology. Qual. Res. Psychol. 3:77-101.

Canadian Radio-television and Telecommunications Commission. 2015. Communications Monitoring Report 2015: Canada's Communications System: An Overview for Citizens, Consumers, and Creators. Accessed Aug. 11, 2017. http://www.crtc.gc.ca/eng/ publications/reports/policymonitoring/2015/cmr2.htm.

Dairy Farmers of Canada. 2015. proAction: Leading the way for sustainable dairy farming; providing assurance to customers about farm practices. Dairy Farmers of Canada, Ottawa, Ontario, Canada. Accessed Dec. 18, 2016. https://www.dairyfarmers.ca/Media/ Files/proaction/proaction_ang_lr15.pdf.

Faulkner, P. M., and D. M. Weary. 2000. Reducing pain after dehorning in dairy calves. J. Dairy Sci. 83:2037-2041. 
Heinrich, A., T. F. Duffield, K. D. Lissemore, and S. T. Millman. 2010. The effect of meloxicam on behavior and pain sensitivity of dairy calves following cautery dehorning with a local anesthetic. J. Dairy Sci. 93:2450-2457.

Milligan, B. N., T. F. Duffield, and K. D. Lissemore. 2004. The utility of ketoprofen for alleviating pain following dehorning in young calves. Can. Vet. J. 45:140-143.

Ritter, C., J. Jansen, S. Roche, D. F. Kelton, C. L. Adams, K. Orsel, R. J. Erskine, G. Benedictus, T. J. G. M. Lam, and H. W. Barkema. 2017. Invited review: Determinants of farmers' adoption of management-based strategies for infectious disease prevention and control. J. Dairy Sci. 100:3329-3347.

Ritter, C., J. Jansen, K. Roth, J. P. Kastelic, C. L. Adams, and H. W. Barkema. 2016. Dairy farmers' perceptions towards the implementation of on-farm Johne's disease prevention and control strategies. J. Dairy Sci. 99:9114-9125.

Robbins, J. A., D. M. Weary, C. A. Schuppli, and M. A. G. von Keyserlingk. 2015. Stakeholder views on treating pain due to dehorning dairy calves. Anim. Welf. 24:399-406.

Stafford, K. J., and D. J. Mellor. 2011. Addressing the pain associated with disbudding and dehorning in cattle. Appl. Anim. Behav. Sci. $135: 226-231$
Stock, M. L., S. L. Baldridge, D. Griffin, and J. F. Coetzee. 2013. Bovine dehorning: Assessing pain and providing analgesic management. Vet. Clin. North. Am. Food Anim. 29:103-133.

Vasseur, E., F. Borderas, R. I. Cue, D. Lefebvre, D. Pellerin, J. Rushen, K. M. Wade, and A. M. de Passille. 2010. A survey of dairy calf management practices in Canada that affect animal welfare. J. Dairy Sci. 93:1307-1315.

Ventura, B. A., M. A. G. von Keyserlingk, and D. M. Weary. 2015. Animal welfare concerns and values of stakeholders within the dairy industry. J. Agric. Environ. Ethics 28:109-126.

Winder, C. B., S. J. LeBlanc, D. B. Haley, K. D. Lissemore, M. A Godkin, and T. F. Duffield. 2016. Practices for the disbudding and dehorning of dairy calves by veterinarians and dairy producers in Ontario, Canada. J. Dairy Sci. 99:10161-10173.

Winder, C. B., S. J. LeBlanc, D. B. Haley, K. D. Lissemore, M. A. Godkin, and T. F. Duffield. 2017. Comparison of an online learning module to hands-on training in teaching cautery disbudding technique for dairy calves, including cornual nerve block application. Can. Vet. J. 58:735-740. 